\title{
Standardised quality control and quality assurance activities in radiology across Australia and New Zealand: in search of the Holy Grail
}

\section{Amanda Perdomo ${ }^{1}$}

Published online: 8 November 2018

(c) Australasian College of Physical Scientists and Engineers in Medicine 2018

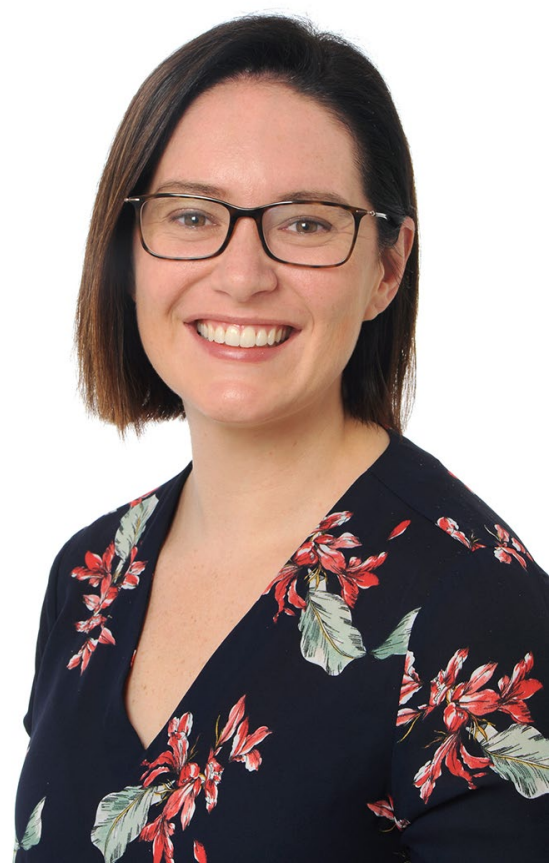

Across Australia and New Zealand, radiation use in medicine is regulated by differing governing bodies in each State and Territory. Although most heavily rely on the Australian Radiation Protection and Nuclear Safety Agency (ARPANSA) Codes of Practice, this is a broad guidance and the details of implementation vary between jurisdictions. This leads fundamentally to considerable differences in requirements for reporting, shielding design, quality assurance (QA) and quality control (QC). Additionally,

Amanda Perdomo

Amanda.Perdomo@rch.org.au

1 ACPSEM Radiology Specialty Group, The Royal Children's Hospital Melbourne, Melbourne, VIC, Australia multiple radiation safety standards applicable to radiology are currently in-force across jurisdictions based on outdated technology, with QC standards that have been well outpaced by advances in imaging equipment technology. This leads to Diagnostic Imaging Medical Physicists (DIMPs), an underresourced profession, spending time on regulated QA and QC activities that could justifiably be considered futile and ineffective.

The American Association of Physicists in Medicine (AAPM) statement on the scope of practice of clinical medical physics says "the essential responsibility of the Qualified Medical Physicist's clinical practice is to assure the safe and effective delivery of radiation. The medical physicist performs or supervises the technical aspects of procedures necessary to achieve this objective. The responsibilities of the medical physicist include the development and direction of quality assurance programs." [1]

Some of the specifics of practice for a DIMP include [1]:

- Develop specifications for imaging equipment

- Develop procedures for the initial and continuing evaluation of imaging equipment

- Measure and characterize the medical radiation from imaging equipment prior to clinical utilization

- Perform acceptance testing, evaluation and commissioning of imaging equipment.

It is widely accepted in the medical community that DIMPs are the experts in the QA and QC required for ionising radiation imaging equipment. However, in Australia and New Zealand, the ACPSEM have not had a strong voice in providing guidance, opinions or control in this area of our practice. This has also contributed to the disparity in regulatory requirements for QA and QC across different jurisdictions.

Currently, each jurisdiction has its own set of mandated requirements for testing. These vary greatly, from the tests 
performed, the compliance criteria as well as varying frequencies (see Table 1):

Almost 12 months ago, the Radiology specialty group (SG) of the ACPSEM decided that it was finally time to take responsibility of these professional activities and attempt to standardise the required minimum QA and QC activities performed on radiology equipment across Australia and New Zealand.

Members of the ACPSEM have previously been responsible for an in-depth ACPSEM position paper for mammography Recommendations for a Digital Mammography QA Program [2]. This position paper has been instrumental in establishing standardised QA and QC across Australia and New Zealand. However, for all other radiology modalities, there has not been the same traction and the mandated QA and QC tests have been developed by each individual state regulator.

With a view to setting a goal achievable in the medium term, the Radiology SG established two working parties: The Angiography and Fluoroscopy QA and QC Working Group and The General X-ray QA and QC Working Group. Each with the aim to develop a set of recommendations for QA and QC for the respective modalities as an extension to the ACPSEM position paper Recommendations for a technical quality control program for diagnostic $X$-ray equipment [3]. These position papers will outline recommendations on QA and QC activities that should be performed on these units, the frequency that they should be performed, which professional craft group should be performing the activity and the recommended threshold for compliance and optimum performance.

The ultimate aim of these position papers is that they are adopted by each state regulator, The Royal Australian and New Zealand College of Radiologists (RANZCR), Diagnostic imaging Accreditation Scheme (DIAS) etc. to develop a standardised approach to QA and QC across Australia and New Zealand.
The two working parties each have representatives from New Zealand, Queensland, New South Wales Victoria and ARPANSA. Each party meets via teleconference approximately every four weeks to discuss the proposed QA and QC activities. These conversations have led to some robust discussions from a difference of opinions as well as exposure to different ideas from people with a range of clinical experience and that trained in different centres across Australia and New Zealand.

One of the main concerns from the working party members was that the time and effort being put into these position papers would be futile as there was no certainty that these will be adopted by regulators and governing bodies. However, the timing of these working groups was serendipitous in that it coincides with work being done by the Radiation Regulators Network (RRN), a collaboration of radiation regulators from all jurisdictions across Australia, also having established a working party to work on the standardisation of requirements for diagnostic imaging equipment. Discussions with the RRN allowed each of the working parties to establish a more defined remit for the work they are trying to produce. Mainly, the RRN agreed that the ACPSEM is well placed to develop ongoing QA and QC recommendations and procedures which, if suitable for the RRN, could be adopted as the expected standard by regulators. The RRN noted and encouraged the work of the ACPSEM working parties and would be keen to adopt our recommendations in their respective states. It seems that we may be closer to reaching our final aim than we expected!

It should also be noted that the two-way conversation between the ACPSEM and the RRN will only improve our practice, working relationships with the regulators and other governing bodies and ultimately, outcomes for our patients. The RRN have also requested several other topics, such as shielding in Radiology, that they would like ACPSEM to publish a position paper on. The communication channel between the ACPSEM and RRN will allow the ACPSEM Radiology SG to develop position papers for areas of interest

Table 1 Mandated compliance testing frequencies, in years, for each jurisdiction in Australia and New Zealand

\begin{tabular}{|c|c|c|c|c|c|c|}
\hline & X-ray & Fluoroscopy & CT & Mammography & OPG/dental & DEXA \\
\hline Queensland & 3 & 1 & 1 & 1 & 3 & NA \\
\hline New South Wales & 5 & 2 & 2 & 2 (1 with MGD) & 5 & 5 \\
\hline Australian Capital Territory & \multicolumn{6}{|c|}{ After new installation or major repair } \\
\hline Victoria & 2 & 2 & 1 & 1 (film-screen) & NA & NA \\
\hline Tasmania & 2 & 2 & 2 & 1 & 4 & 4 \\
\hline South Australia & \multicolumn{6}{|c|}{ After new installation or major repair } \\
\hline Western Australia & 2 & $21(\mathrm{C}-\mathrm{arm})$ & 2 & 1 & 3 & NA \\
\hline Northern Territory & 1 & 1 & 1 & 1 & 2 & NA \\
\hline New Zealand & 1 (busy centre) 2 & 1 & 1 & 1 & $\begin{array}{c}2 \text { (OPG/cone beam) } \\
\text { NA (intra-oral) }\end{array}$ & $2-3$ \\
\hline
\end{tabular}


for the regulators to continuously improve our multidisciplinary approach to improving patient outcomes. With the recent restructure of the ACPSEM SGs including the introduction of executive committees, it may be possible to have multiple working groups on differing areas of interest to produce a number of position papers simultaneously. This will allow us to keep ahead, or up to date, with technological changes.

My initial aim was to use this editorial to announce that there were two brand new shiny documents for all Radiology medical physicists to take to their departments to implement so we could all have a standardised practice. However, like most things, time lines blow out especially when it is dependent on a dedicated group of volunteers, all who have actual paid jobs to be doing. So, instead I am updating you on the hard work of the volunteers in both these working parties and a promise that the documents will be landing in your inbox as soon as possible. Nonetheless, the advanced development of the documents themselves and more importantly the positive steps to maintaining a consistent framework in collaboration with the RRN is a significant advancement in the right direction. The quest continues.

\section{References}

1. https://www.aapm.org/org/policies/details.asp?id=317\&type=PP\&curre $\mathrm{nt}=$ true

2. Heggie JCP, Barnes P, Cartwright L, Diffey J, Tse J, Herley J, McLean ID, Thomson FJ, Grewal RK, Collins LT (2017) ACPSEM position paper: digital mammography V4.0. Australas Phys Eng Sci Med 40:491. https://doi.org/10.1007/s1324 6-017-0583-x

3. Causer DA, Einsiedel P, Heggie P, Wallace JC, Schick A, Grewal D, Collins RK, Coakley L, Hill K, McLean B, Atkinson ID, J. ACPSEM (2005) Position Paper: recommendations for a technical quality control program for diagnostic X-ray equipment Australas. Phys Eng Sci Med 28:69. https://doi.org/10.1007/BF03178696 